\title{
PREVALENCIA DE DISPLASIA CERVICAL EN EMBARAZADAS EN EL HOSPITAL UNIVERSITARIO DE MONTERREY, NUEVO LEÓN, MÉXICO.
}

\author{
Clara del Carmen Flores Acosta, Oscar Vidal Gutierrez, Donato Saldívar Rodríguez, \\ Rey David Núñez Alvar.
}

Departamento de Ginecología y Obstetricia, Hospital Universitario “Dr. José Eleuterio González”, Monterrey, Nuevo León, México.

\section{RESUMEN}

Objetivo. Determinar la prevalencia de displasia cervical en mujeres embarazadas. Método. Estudio observacional, longitudinal, prospectivo, en el cual se analizaron los resultados de 250 pacientes embarazadas que acudieron a control prenatal en el Hospital Universitario "Dr. José Eleuterio González", Monterrey, Nuevo León, México, en el período enero 2007 a julio de 2008. Resultados. Se analizaron 36 citologías anormales $(14,4 \%)$, las cuales reportaron ASCUS 3,2\% $(n=8)$, LIEBG $8 \%(n=20)$, LIEAG $2,4 \%(n=6)$ y CaCu $0,8 \%(n=2)$. La prevalencia de displasia cervical confirmada por biopsia fue $5,6 \%(n=14)$. Conclusiones. Se recomienda realizar la citología cervical a toda paciente embarazada, para la detección y tratamiento oportuno de las displasias cervicales.

\section{PALABRAS CLAVE: Citología cervical, displasias cervicales, cáncer cérvicouterino, embarazo, control prenatal}

\section{SUMMARY}

Objective. To evaluate the prevalence of cervical dysplasia among pregnant women. Method. We conducted an observational, longitudinal, prospective study, that analyzed the results of 250 cervical smears of pregnant patients that attended the Obstetrics consult of the University Hospital in Monterrey, Mexico, between January 2007 and July 2008. Results: 36 abnormal smears were analyzed (14.4\%). Showing ASCUS in $3.2 \%(n=8)$, LSIL in $8 \%(n=20)$, HSIL in $2.4 \%(n=6)$ and AIS in $0.8 \%(n=2)$. The prevalence of cervical dysplasia confirmed by biopsy was $5.6 \%(n=14)$. Conclusions: We recommend performing cervical smears to all pregnant women for detection and appropriate treatment of cervical dysplasia.

KEY WORDS: Cervical cytology, cervical dysplasia, cervical cancer, pregnancy, prenatal care

\section{INTRODUCCIÓN}

El cáncer cérvico-uterino $(\mathrm{CaCu})$ representa la primera causa de muerte en mujeres mayores de 25 años en México (1). Aproximadamente el 30\% de las mujeres diagnosticadas se encuentran en etapa reproductiva y el $3 \%$ se encuentran emba- razadas o en el periodo posparto al momento del diagnóstico (2). Se considera una de las neoplasias malignas más comunes en el embarazo, con una incidencia estimada de 0,8 a 1,5 casos por 10.000 nacimientos. La mayoría de los casos se identifican como resultado de los programas de detección (3).

Durante el embarazo la incidencia promedio de 
citologías cervicales anormales es de 5 a $8 \%$; el de carcinoma in situ de 1,3 por 1000 y la de carcinoma invasor es 1 cada 2.200 embarazos $(4,5)$. El pico máximo de incidencia del carcinoma cervical in situ ocurre entre los 25 y 35 años, mientras que la incidencia del $\mathrm{CaCu}$ aumenta después de los 40 años $(6,7)$.

Se ha observado que tanto el estadio, el curso y el pronóstico del $\mathrm{CaCu}$ en pacientes embarazadas son similares a las no embarazadas $(4,8)$. Desde la introducción de la colposcopia en 1970, como técnica diagnóstica para identificar el probable sitio de lesión de una citología anormal, el manejo de mujeres embarazada ha cambiado, permitiendo realizar tratamientos conservadores. Ya que el tratamiento de lesiones preinvasivas puede ser pospuesto hasta el periodo posparto. La habilidad de distinguir entre una neoplasia intraepitelial cervical y el cáncer invasor ha sido el mejor paso para el manejo de pacientes durante el embarazo $(4,9)$.

El objetivo de este estudio fue determinar la prevalencia de displasia cervical, el manejo y tratamiento en mujeres embarazadas.

\section{PACIENTES Y MÉTODOS}

Se realizó un estudio observacional, prospectivo, longitudinal, en el cuál se incluyeron las pacientes embarazadas que acudieron a la consulta de obstetricia del Hospital Universitario "Dr. José Eleuterio González", en Monterrey, Nuevo León, de enero de 2007 a julio de 2008; para control prenatal con adecuado seguimiento en caso de diagnosticar algún tipo de displasia cervical. A todas las pacientes en la primera visita se les tomó muestra para citología cervical. Se realizó colposcopia y biopsia en aquellas pacientes con citología anormal y a las 6 semanas del puerperio. Los factores de riesgo analizados fueron: edad, inicio de vida sexual activa, número de compañeros sexuales, número de gestaciones, edad del primer embarazo, antecedente de displasia cervical, enfermedades de transmisión sexual, tabaquismo, enfermedades concurrentes, método de planificación familiar.

Se realizó un análisis estadístico descriptivo para las variables sociodemográficas. Los resultados se expresan como promedios \pm desviación estándar $( \pm \mathrm{DE})$. Para la relación de las variables de estudio (factores de riesgo) y la presencia de displasias cervicales se realizó la prueba de chi cuadrado $\left(x^{2}\right)$. Se consideró como significativo un valor de $p<0,05$, para el análisis se utilizó el programa estadístico SPSS versión 8.0.

\section{RESULTADOS}

Se analizaron 250 citologías de pacientes embarazadas con adecuado control prenatal en el servicio de Obstetricia del Hospital Universitario "Dr. José Eleuterio González". La toma de citología cervical fue realizada en el primer trimestre en el $8 \%$ $(n=20)$, en el segundo el $88 \%(n=220)$ y el $4 \%$ en el tercero $(n=10)$. Hubo 36 casos $(14,4 \%)$ de pacientes con citología cervical anormal a las cuales se les realizó colposcopia y biopsia; confirmando el diagnóstico en $14(5,6 \%)$, de las cuales 8 presentaban lesión intraepitelial de bajo grado (LIEBG), 4 lesión intraepitelial de alto grado (LIEAG) y $2 \mathrm{CaCu}$ invasor (Tabla I).

Tabla I

\section{RESULTADOS DE LAS CITOLOGÍAS Y BIOPSIAS CERVICALES EN LAS PACIENTES EMBARAZADAS}

\begin{tabular}{lccc}
\hline $\begin{array}{l}\text { Citología } \\
\text { cervical }\end{array}$ & $\begin{array}{c}\text { Citología } \\
(\mathrm{n}=250)\end{array}$ & $\begin{array}{c}\text { Colposcopia } \\
\text { y biopsia } \\
(\mathrm{n}=36)\end{array}$ & $\begin{array}{c}\text { Colposcopia } \\
\text { y biopsia en } \\
\text { puerperio } \\
(\mathrm{n}=34)\end{array}$ \\
\hline Normal & $214(85,6 \%)$ & 22 & 27 \\
LIEBG & $20(8 \%)$ & $8(3,2 \%)$ & 3 \\
LIEAG & $6(2,4 \%)$ & $4(1,6 \%)$ & 4 \\
ASCUS & $8(3,2 \%)$ & 0 & - \\
CaCu & $2(0,8 \%)$ & $2(0,8 \%)$ & - \\
\hline
\end{tabular}

LIEBG: lesión intraepitelial de bajo grado. LIEAG: lesión intraepitelial de alto grado. ASCUS: Atypical Squamous Cells of Undetermined Significance. CaCu: cáncer cérvicouterino.

En cuanto a las variables sociodemográficas observamos que la edad promedio de las pacientes fue de $22,8 \pm 4,15$ años. El $50 \%$ de las pacientes era menor de 21 años. El $25 \%$ de las pacientes que resultaron con alteración se habían realizado citología anteriormente y solo el $14 \%$ con resultados normales. La presencia de tabaquismo activo (5 o más cigarros/día) se observó en el $67 \%$ de las pacientes con displasia. No se observo diferencias estadísticamente significativas entre los factores de riesgo evaluados y la presencia de displasia cervical. Los factores de riesgo analizados se observan en la Tabla II.

La vía de nacimiento en el grupo de pacientes con citología anormal fue vaginal en 23 casos 


\section{Tabla II}

CARACTERÍSTICAS Y FACTORES DE RIESGO EN PACIENTES EMBARAZADAS

\begin{tabular}{lccc}
\hline & $\begin{array}{c}\text { Pacientes sin displasia } \\
(\mathrm{n}=214)\end{array}$ & $\begin{array}{c}\text { Pacientes con displasia } \\
(\mathrm{n}=36)\end{array}$ & Valor $\mathrm{p}^{*}$ \\
\hline Edad (años) & $22,8 \pm 4,1$ & $23,1 \pm 5,3$ & 0,24 \\
Número de embarazos & $1,7 \pm 0,7$ & $2,1 \pm 0,8$ & 0,14 \\
Edad de inicio de vida sexual & $19,2 \pm 2,3$ & $18,1 \pm 2,1$ & 0,7 \\
Número de compañeros sexuales & $2,1 \pm 0,4$ & $2,6 \pm 0,8$ & 0,21 \\
Enfermedad de transmisión sexual & $1(0,4 \%)$ & 0 & 0,32 \\
Método de planificación familiar & $7(3,2 \%)$ & $2(5,5 \%)$ & 0,4 \\
Tabaquismo & $102(48 \%)$ & $24(67 \%)$ & 0,89 \\
Citología cervical previo al embarazo & $54(25 \%)$ & $5(14 \%)$ & - \\
\hline
\end{tabular}

* No significativo

$(63,8 \%)$ y $13(36,2 \%)$ por cesárea, 2 de las cuales fueron por $\mathrm{CaCu}$ invasor y el resto por alguna indicación obstétrica.

Las pacientes con citología anormal fueron valoradas en el periodo posparto (a las 6 semanas) con citología y biopsia. Del grupo de pacientes con displasia cervical $(n=12)$ se observó regresión en 5 casos $(41,6 \%)$ y persistencia en $7(58,3 \%)$. El tratamiento utilizado para estas pacientes fue escisión con asa de diatermia en LIEBG, conización en LIE$A G$. En los casos de $\mathrm{CaCu}$ invasor $(n=2)$ se realizó cirugía radical de Wertheim.

\section{DISCUSIÓN}

El embarazo representa una oportunidad excepcional para del diagnóstico temprano del cáncer cervicouterino desde que la inspección visual, la realización de citología cervical y la palpación bimanual se han considerado como parte de la valoración rutinaria del control prenatal $(3,10)$.

La prevalencia de citologías cervicales anormales durante el embarazo detectada en nuestro estudio fue del $14,4 \%$, siendo mayor a lo reportado en la literatura $(5-8 \%)$, así como la incidencia de $\mathrm{CaCu}$ invasor $0,8 \%(0,1-0,02 \%)(7,11)$. Existen reportes de que las displasias diagnosticadas durante el embarazo, presentan regresión entre el 10$70 \%$, la persistencia de la severidad se reporta en el $25-47 \%$ y la progresión ocurre en el 3-30\% de los casos $(4,11)$. Nosotros observamos regresión en el $41,6 \%$ de los casos y persistencia en el $58,3 \%$.

Los resultados analizados en este estudio apoyan las recomendaciones realizadas en cuanto a que la citología cervical constituye una prueba de tamizaje que debe ser una práctica obligada que forme parte del cuidado prenatal. Las intervencio- nes diagnósticas como la colposcopia deben realizarse por personal altamente capacitado, ya que los cambios fisiológicos asociados al embarazo pueden dificultar el diagnóstico $(4,8)$ La biopsia de lesiones sospechosas es una intervención necesaria y aceptable y debe realizarse bajo visualización colposcópica. El inicio del tratamiento del carcinoma invasor del cérvix debe basarse en la edad gestacional al momento del diagnóstico $(7,9,10)$.

\section{CONCLUSIÓN}

Se recomienda realizar la citología cervical a toda paciente embarazada, para la detección y tratamiento oportuno de las displasias cervicales.

\section{BIBLIOGRAFÍA}

1. Sosa-Rubi SG, Walker D, Serván E. Práctica de mastografías y pruebas de Papanicolaou entre mujeres de áreas rurales de México. Salud Publica Mex 2009;51 supl 2:S236-S45.

2. Flannelly $\mathrm{G}$. The management of women with abnormal cervical cytology in pregnancy. Best Pract Res Clin Obstet Gynaecol 2010;24(1):51-60.

3. Siddiq TS, Tiwigg JP, Hammond RH. Assessing the accuracy of colposcopy at predicting the outcome of abnormal cytology in pregnancy. Eur J Obstet Gynecol Reprod Biol 2006;124(1):93-7.

4. Van Calsteren K, Vergote I, Amant F. Cervical neoplasia during pregnancy: Diagnosis, management and prognosis. Best Pract Clin Obstet Gynaecol 2005;19(4):611-30.

5. Castro P, Yazigi R, Prado S, Garrido J. Cáncer cervicouterino y embarazo: experiencia del Instituto Nacional del Cáncer. Rev Chil Obstet Ginecol 2004;69(4):274-8. 
6. Oduncu FS, Kimmig R, Hepp H, Emmerich B. Cancer in pregnancy: maternal-fetal conflict. J Cancer Res Clin Oncol 2003;129(3):133-46.

7. Hunter MI, Monk BJ, Tewari KS. Cervical neoplasia in pregnancy. Part 1: screening and management of preinvasive disease. Am J Obstet Gynecol 2008;199(1):3-9.

8. Sarkar S, Yusif S, Egan D. Cervical screening during pregnancy. Ir Med J 2006;99(9):284-5.

9. Vlahos G, Rodolakis A, Diakomanolis E. Conservati- ve management of cervical intraepithelial neoplasia $(\mathrm{CIN}(2-3))$ in pregnant women. Gynecol Obstet Invest 2002;54(2):78-81.

10. Siddiq TS, Twigg JP, Hammond RH. Assessing the accuracy of colposcopy at predicting the outcome of abnormal cytology in pregnancy. Eur J Obstet Gynecol Reprod Biol 2006;124(1):93-7.

11. Boardman LA, Goldman DL, Cooper AS. CIN in pregnancy: antepartum and postpartum cytology and histology. J Repro Med 2005;50(1):13-8. 